\title{
Ten minute radiopharmaceutical test in biliary atresia
}

\author{
M A EL TUMI, M B CLARKE, J J BARRETT, AND A P MOWAT \\ Departments of Child Health and Nuclear Medicine, King's College Hospital, London
}

SUMmARY To provide an objective rapid means of excluding extrahepatic biliary atresia (atresia), a hepatic index was devised from the ratio of the net hepatic to cardiac distribution of ${ }^{99 m}$ Tc diisopropyl iminodiacetic acid or methylbrom iminodiacetic acid between 2.5 and 10 minutes after injection. The hepatic index was compared with subjective assessment of abdominal scintigraphy performed repeatedly over 24 hours. In 22 infants with hepatitis the hepatic index ranged from 5.03 to 14.9 , one having no excretion on scintiscan. In 26 infants with atresia the index ranged from 0.49 to 4.26 and in two with paucity of intralobular bile ducts it was 1.85 and 3.69. None of these infants had excretion apparent on scintiscans. Similarly, low hepatic indices occurred in four infants with liver dysfunction but pigmented stools, three of whom had no excretion apparent on scintiscans. These preliminary studies suggest that a hepatic index of greater than 5 is much more rapid and as specific in excluding atresia as repeated abdominal scintigraphy.

Over $70 \%$ of infants with extrahepatic biliary atresia (atresia) become free of jaundice after portoenterostomy if the operation is performed in the first eight weeks of life. ${ }^{1}$ Ninety per cent of those who lose their jaundice are thriving at 10 years of age. ${ }^{2}$ Accurate pre-laparotomy differentiation of atresia from intrahepatic disorders that cause severe cholestasis is essential if the correct infants are to be selected for this radical operation. Findings on laparotomy may be misleading in up to $20 \%$ of infants, with the danger of inappropriate removal of small, narrow, but patent bile ducts in infants with intrahepatic disease. ${ }^{34}$ Radiopharmaceuticals, which are preferentially excreted into the biliary system and thereby show bile duct patency, provide important information for identifying infants who do not require laparotomy.

Recently, abdominal scintigraphy after intravenous administration of ${ }^{9 \% \mathrm{~m}}$ technetium diisopropyl iminodiacetic acid (DISIDA) in infants pretreated with phenobarbitone has replaced the ${ }^{131} \mathrm{I}$ Rose-Bengal faecal excretion test as it is as accurate and much more convenient. ${ }^{5}$ There are three major problems in interpreting abdominal scintiscans. Firstly, the decision whether the isotope is in the kidneys or gall bladder or in the urinary bladder or bowel is entirely subjective. Secondly, in those infants with severe intrahepatic cholestasis and slow excretion the radiopharmaceutical may only enter the bowel after 12,18 , or 24 hours, by which time it is often not detected as most of the radionuclide has decayed. Thirdly, the test is also time consuming as it requires repeated scans at intervals from 20 minutes after injection up to 24 hours. To overcome these difficulties we have developed a technique that is objective, avoids confusion caused by urinary excretion of the isotope, and is based on the tissue distribution of the radiopharmaceutical in the first 10 minutes after intravenous injection.

\section{Patients}

A total of 54 infants with jaundice aged 3-16 weeks were studied between December 1983 and December 1985 . Fifty infants aged 3-12 weeks had acholic stools and were considered to be possible cases of atresia. Final diagnoses were: 26 cases of atresia, 17 of idiopathic hepatitis of infancy, five of $\alpha_{1}$ antitrypsin deficiency, and two of paucity of the interlobular bile ducts (biliary hypoplasia). The diagnosis of atresia was confirmed by histological examination of the biliary remnants removed at portoenterostomy. $\alpha_{1}$ Antitrypsin phenotyping was determined by isoelectric focusing. ${ }^{6}$ Diagnosis in other cases was made by excluding known causes of liver damage in this age group, by histological assessment of percutaneous liver biopsy specimens, and by the patient's subsequent course.

At the time of study the mean values of standard biochemical tests of liver function, serum bilirubin 
concentration, aspartate aminotransferase, $\gamma$-glutamyltranspeptidase, and alkaline phosphatase activities were not significantly different in those with atresia and those with intrahepatic disease.

To investigate the specificity of our findings and clarify mechanisms responsible the investigation was performed in four further infants who had bile pigmented stools and thus were not suspected of having atresia. Two had idiopathic fulminant hepatic failure, one was recovering from portal pyaemia, and one had liver disease associated with panhypopituitarism. Informed consent was obtained from the parents.

\section{Methods}

Development of the hepatic index. After at least three days of oral phenobarbitone in a dose of 5 $\mathrm{mg} / \mathrm{kg} / \mathrm{day}^{8} 37 \mathrm{mBq}$ of ${ }^{99 \mathrm{~m}} \mathrm{Tc}$ DISIDA (Disofen, New England Nuclear Chemicals Ltd) was given intravenously. The infant was placed for 10 minutes over an International General Electric (535) gammacamera linked to an Analog Data Analysis Company computer system. Gamma emissions were recorded continuously in 30 second frames.

One 30 second recording was subsequently displayed on a television monitor and, using the computer linked pen, areas were defined within the outline of the liver, heart, and lung fields (representing background activity) (Fig. 1). The counts in these areas were analysed in all 30 second frames, corrected to unit area, and depicted as an activity/time curve, giving the count density over the liver, heart, and background as a function of time. Analysis of these curves in 19 infants, 11 of whom had atresia, showed that complete separation of patients with atresia from those with intrahepatic disease could be obtained from frames between 2.5 and 10 minutes after injection on the basis of the ratio of the net counts over the liver and heart. This

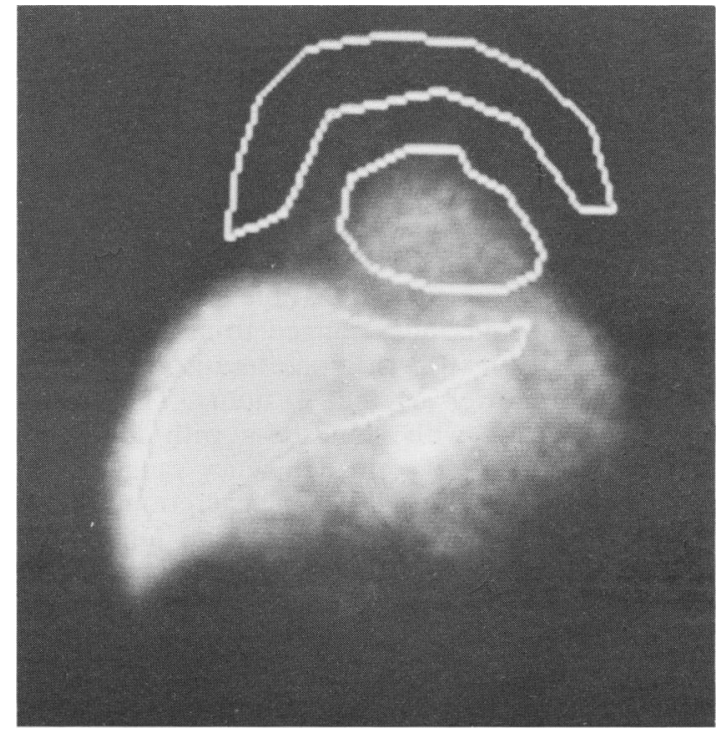

Fig. 1 Black and white photograph of the representation in colour on the video screen of the gamma irradiation in one 30 second frame: Areas of interest within the outlines of the liver and heart and an area encircling the heart representing background activity have been drawn with a computer linked light pen.

ratio, counts over liver minus counts over background divided by counts over heart minus counts over background during this 7.5 minute period, we termed the hepatic index.

Prospective study. The hepatic index was applied prospectively in 35 infants. In these studies the derivative of iminodiacetic acid ${ }^{99} \mathrm{~m}$ technetium trimethylbrom-IDA (TMB-IDA) (Sorin Biomedica SPA) was used as DISIDA was withdrawn temporarily from use in the United Kingdom. TMB-IDA

Table Hepatic index and subjective assessment of isotope excretion in abdominal scintiscans after intravenous ${ }^{99 m}$ technetium iminodiacetic acid (DISIDA and TMB-IDA)

\begin{tabular}{|c|c|c|c|c|c|}
\hline \multirow[t]{2}{*}{ Disorder } & \multirow[t]{2}{*}{$\begin{array}{l}\text { No of } \\
\text { cases }\end{array}$} & \multicolumn{2}{|c|}{ Hepatic index } & \multicolumn{2}{|c|}{$\begin{array}{l}\text { Scintigraphic findings of } \\
\text { intestinal radioactivity }\end{array}$} \\
\hline & & Range & Mean $(S D)$ & Absent & Present \\
\hline Biliary atresia & 26 & $0 \cdot 49-4 \cdot 26$ & $2 \cdot 59(0 \cdot 19)$ & 26 & - \\
\hline Idiopathic hepatitis of infancy & 17 & $5 \cdot 03-12 \cdot 27$ & $8 \cdot 31(0 \cdot 62)$ & 1 & 16 \\
\hline Hepatitis with $\alpha_{1}$ antitrypsin deficiency & 5 & $7 \cdot 2 \cdot-14 \cdot 19$ & $9.8 \quad(1.4)$ & - & 5 \\
\hline Paucity of interlobular bile ducts & 2 & 1.85 & & 1 & - \\
\hline \multirow[t]{2}{*}{ Fulminant hepatic failure } & 2 & $0 \cdot 80$ & & 1 & - \\
\hline & & $3 \cdot 76$ & & 1 & - \\
\hline Portal pyaemia & 1 & $2 \cdot 30$ & & - & 1 \\
\hline Panhypopituitarism & 1 & $1 \cdot 34$ & & 1 & - \\
\hline
\end{tabular}


had the advantage of improved hepatic uptake and less renal excretion. ${ }^{9}$

\section{Results}

The hepatic index and the results of the conventional 24 hour ${ }^{99} \mathrm{~m}_{\text {technetium iminodiacetic acid }}$ (DISIDA and TMB-IDA) abdominal scintigraphy in all 54 infants are given the Table. In patients with atresia the hepatic index ranged from 0.49 to 4.26 (mean (SD) $2.59(0 \cdot 19)$ ). None had isotope excretion into the bowels even at 24 hours. The hepatic index in infants with cholestasis with idiopathic hepatitis or $\alpha_{1}$ antitrypsin deficiency ranged from 5.03 to $14 \cdot 19$. All showed isotope excretion into the bowels within four hours except one infant with idiopathic hepatitis in whom no excretion occurred even at 24 hours. The hepatic index in this infant was 5.03. No isotopic activity was detected in the bowels of two infants with paucity of intrahepatic bile ducts. Their hepatic indices were 1.85 and 3.69.

Two infants with fulminant hepatic failure and one with panhypopituitarism also had low hepatic indices $(1.85,3.69$, and 1.34 , respectively). No bowel radioactivity could be detected in these infants and all had markedly reduced hepatic uptake. The infant recovering from portal pyaemia also had a low hepatic index of $2 \cdot 3$ but bowel radioactivity was evident at seven hours. This infant, too, had markedly reduced hepatic uptake. The comparison of the hepatic index and hepatic uptake (Fig. 2) showed that when the index was greater than 5 there was a high uptake of the radiopharmaceutical, whereas when the index was less than 5

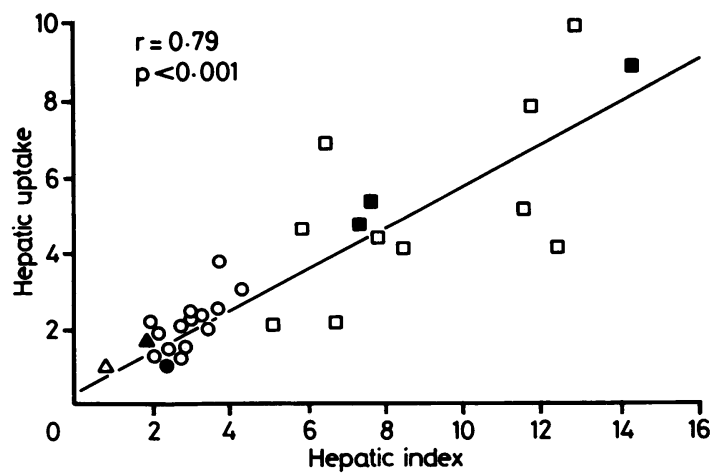

Fig. 2 The relation between hepatic index and hepatic uptake (the ratio of net hepatic counts to background counts) in 30 infants. $\bigcirc=$ Biliary atresia; $\square=$ idiopathic hepatitis of infancy; $=$ hepatitis with $\alpha_{1}$ antitrypsin deficiency; $=$ portal pyaemia; $\Delta=$ fulminant hepatic failure; $\boldsymbol{\Delta}=$ paucity of intrahepatic bile ducts. the uptake was reduced. There was no significant relation beween the hepatic index and net cardiac counts.

\section{Discussion}

Without effective surgery the mean age at death of patients with atresia is 11 months. ${ }^{10}$ Portoenterostomy by an experienced surgeon produces bile drainage in up to $90 \%$ of infants if performed by 8 weeks of age $(90 \%$ of those free of jaundice remain well at 10 years ${ }^{1}$ ) and survival until the third decade has been documented. ${ }^{11}$ In contrast, infants with intrahepatic disease have a much better natural history, often making a complete recovery unless associated with genetic or familial disorders or paucity of the intrahepatic bile ducts. ${ }^{12}{ }^{13}$ If, however, such infants with complete cholestasis are submitted to laparotomy and cholangiography, even by experienced surgeons, inappropriate radical surgical procedures may be performed. ${ }^{4}$ It has been our practice to submit to laparotomy infants with histological features in percutaneous liver biopsy specimens that are indicative of bile duct obstruction, unless the patient has been shown to have some genetic abnormality, such as $\alpha_{1}$ antitrypsin deficiency or cystic fibrosis, or if syndromic paucity of the interlobular bile ducts or bile duct patency has been shown with radiopharmaceuticals. ${ }^{7}$ In a recent study of 32 infants with severe cholestasis we have found that DISIDA scintigraphy performed after treatment with phenobarbitone was as efficient in showing bile duct patency as ${ }^{131}$ I Rose-Bengal faecal excretion. ${ }^{5}$ As the latter test takes at least three days and makes heavy demands on nursing time we no longer use it.

DISIDA and TMB-IDA are rapidly taken up by the liver and have low but appreciable renal excretion in infants with cholestasis. ${ }^{99 \mathrm{~m}}$ Technetium has good imaging properties but its short half life of six hours makes assessment of scintiscans difficult after 12 hours. Other authors have evaluated clearance from the blood and hepatic uptake in an effort to overcome problems caused by renal excretion of the radiopharmaceutical and short half life of ${ }^{99 m}$ technetium.

Gerhold and coworkers, in a study of 16 infants with atresia and 11 with hepatitis using DISIDA and diethyl IDA, used an entirely subjective method of grading clearance from the blood. ${ }^{14}$ Patients with no visible cardiac radioactivity after five minutes had a numerical score of 1 , while if there was considerable retention in the circulation, with the cardiac activity equalling or exceeding the hepatic activity by five minutes, a score of 4 was given. These authors found that patients with atresia had low scores and those 
with hepatitis higher scores but that there was considerable overlap between the two groups.

Kirk and coworkers, in a study of 15 infants with cholestasis using DISIDA, analysed 12 images taken in the first hour and five taken in the subsequent 23 hours. ${ }^{15}$ They found that in infants with hepatitis and severe hepatocellular damage uptake into the liver was delayed, while in infants with atresia the uptake was normal. Again the assessment was subjective.

Bourdelat and coworkers, in a study of eight infants, three of whom had atresia, used diethyl IDA scintographic images to generate time/activity curves. ${ }^{16}$ They also concluded that a good hepatic uptake was suggestive of atresia. The single time/ activity curve given, however, showed that in the early stages the opposite was true. Lennard et al, using diethyl IDA, subjectively analysed time/ activity curves obtained over 60 minutes in six infants with atresia and 11 with hepatitis and found no appreciable hepatic uptake in patients with hepatitis but good hepatic uptake in patients with atresia.

In our studies using both DISIDA and TMB-IDA we found the opposite. In those patients with a cholestatic syndrome that was clinically indistinguishable from atresia the hepatic index was more satisfactory in those with hepatitis than in those with atresia. This may be due to differences in the patient population studied, treatment with phenobarbitone beforehand, or the hepatic extraction of the radiopharmaceuticals used. They are more likely to have arisen, however, because our study concentrated on the very early hepatic uptake and was entirely objective.

The cause of the slow hepatic uptake in patients with atresia is unclear. The clinical and pathological features in infants with hepatocellular damage associated with $\alpha_{1}$ antitrypsin deficiency are often indistinguishable from infants with atresia, but the hepatic index in the patients with $\alpha_{1}$ antitrypsin deficiency was similar to that in other types of intrahepatic disease. The low hepatic indices found in the patients with fulminant hepatic failure with a prothrombin time prolonged by more than 40 seconds at the time of the study was not surprising in that severe hepatocellular failure was present. The low hepatic index found in the patient during the recovery phase was from portal pyaemia and the infant with panhypopituitarism is unexplained.

Knodell and coworkers studied the effects of cholestasis on hepatic uptake of ${ }^{99 \mathrm{~m}}$ technetium $p$-isopropyliminodiacetic acid in isolated rat hepatocytes from animals with bile duct ligation or intrahepatic cholestasis induced by ethinyl oestradiol. ${ }^{18}$ Decreased hepatic uptake occurred in both groups but was more pronounced in those with mechanical obstruction. The authors concluded that the decreased hepatic uptake might be attributable to higher bile salt concentrations but did not in fact measure these.

From the point of view of clinical management, the low hepatic index in the two infants with syndromic paucity of interlobular bile ducts is particularly disappointing as such infants may have severe cholestasis in infancy and some have liver biopsy features compatible with bile duct obstruction in the first six months of life. The non-hepatic features of this syndrome are not sufficiently specific in all cases to make a diagnosis before laparotomy. ${ }^{7}$ At laparotomy the extrahepatic ducts are often so small that cholangiography performed through the gall bladder may fail to fill the intrahepatic ducts. Such infants are at great risk of inappropriate surgery. ${ }^{4}$ These preliminary studies suggest that a hepatic index of 5 or more in this 10 minute investigation is as discriminating and specific in excluding infants with extrahepatic biliary atresia as repeated abdominal scintigraphy over 24 hours. Where the hepatic index is less than 4.3 atresia is a strong possibility but the result must be considered in the light of other clinical and investigative findings.

MAET was supported by a grant from the Secretary of Atomic Energy, Libya.

We thank S Newton and N Monaghan for technical help and Mrs A McPherson for typing the manuscript.

\section{References}

1 McClement JW, Howard ER, Mowat AP. Results of surgical treatment for extrahepatic biliary atresia in United Kingdom 1980-82. Br Med J 1985;290:345-7.

2 Ohi R, Manamatsu M, Mochizuki I, Chiba T, Kasai M. Progress in the treatment of biliary atresia. World J Surg 1985;9:285-93.

3 Hayes DM, Wooley YM, Snyder WH, Reed GD, Gwinn JL, Lending BH. Diagnosis of biliary atresia-relative accuracy of percutaneous liver biopsy, open liver biopsy and operative cholangiography. J Pediatr 1967;71:598-607.

${ }^{4}$ Markowitz J, Daum F, Kahn EI, et al. Arterio hepatic dysplasia. Pitfalls in diagnosis and management. Hepatology 1983;3:74-7.

5 Dick MC, Mowat AP. Biliary scintigraphy with DISIDA. Arch Dis Child 1986;61:191-2.

${ }^{6}$ Frantz RR, Eriksson AW. Reliable classification of six PIM subtypes by separator isoelectric focusing. Hum Hered 1978;28:212-5.

7 Howard ER, Mowat AP. Hepatic biliary disorders in infancy: hepatitis; extrahepatic biliary atresia; intrahepatic biliary hypoplasis. In: Thomas HC, McSween RNM, eds. Recent advances in hepatology. Edinburgh: Churchill Livingstone, 1983:153-69.

${ }^{*}$ Majd M, Reba RC, Altman RP. Effect of phenobarbitone on Tc99m IDA scintigraphy in evaluation of neonatal jaundice. Semin Nucl Med 1981;11:194-204.

9 Nunn AD, Loberg MD, Conley RA. A structure-distributionrelationship approach leading to the development of Tc99m mebrofenin; an improved cholescintigraphic agent. J Nucl Med $1983 ; 24: 423-30$. 
11" Edelman S. Prognosis of uncorrected biliary atresia: an update. J Pediatr Surg 1978;13:389-91.

1 Kasai M. Advances in the treatment of biliary atresia. Jpn J Surg 1983:13:265-70.

12 Dick MC, Mowat AP. Hepatitis syndrome in infancy, an epidemiological survey with 10 year follow-up. Arch Dis Child 1985;60:512-6.

13 Deutsch J, Smith AL, Danks DM, Campbell PE. Long term prognosis for babies with neonatal liver discase. Arch Dis Child 1985;60:447-51.

it Gerhold JP, Klingensmith IIIWC, Kuni CC, et al. Diagnosis of biliary atresia with radionucleotide hepatobiliary imaging. Radiology 1983;146:499-504.

15 Kirk DR, Coleman RE, Filston HC, Rosenberg ER, Merten DF. An imaging approach to persistent neonatal jaundice. AJR 1984;142:461-5.

16 Bourdelat D, Bourguet P, Herry JY, Gruel Y, Guibert L,
Babut JM. Interet de l'exploration scintigraphique au TC99m diethyl-IDA dan le diagnostic de latresie des voies biliaries. Ann Pediatr (Paris) 1983;30:239-44.

17 Lennard JC, Hitch DC, Marion CV. The use of diethyl-IDA $\mathrm{Tc} 99 \mathrm{~m}$ clearance curves in the differentiation of biliary atresia from other forms of neonatal jaundice. Radiology 1982;142: 773-6.

18 Knodell RG, Shafer RB, Stanley LN, Goodman M, Elson MK. Effect of cholestasis on hepatic transport of TC99m p-isopropyliminodiacetic acid. J Lab Clin Med 1984;104: $517-27$.

Correspondence to Dr A P Mowat, Department of Child Health, King's College Hospital, Denmark Hill, London SE5 8RX.

Received 25 September 1986 